\title{
Essais d'amélioration sylvicole de la croissance et de la régénération de taillis traditionnels de bouleau (Betula pubescens Ehrh) et de robinier (Robinia pseudacacia L). Premiers résultats
}

\author{
A Cabanettes*, L Bouvarel, L Pagès** \\ INRA, station de sylviculture et unité d'expérimentation biomasse forestière, \\ Ardon, 45160 Olivet, France
}

(Reçu le 6 novembre 1989; accepté le 14 mars 1990)

\begin{abstract}
Résumé - Deux essais ont été installés en 1982 et 1983 dans 2 taillis simples, de bouleau (Betula pubescens Ehrh) et de robinier (Robinia pseudacacia L), localisés respectivement en Sologne et dans l'Orléanais, dans le but d'améliorer leur croissance et l'efficacité de leur régénération. Les techniques utilisées ont consisté à augmenter l'ensouchement (plantation intercalaire, augmentation des semis de bouleau ou du drageonnement du robinier) et à stimuler la croissance (fertilisation, travail du sol). Les premiers résultats montrent que la réponse des peuplements est relativement limitée, probablement à cause de sols souvent très pauvres, de la compétition juvénile intense et de systèmes racinaires vieillis. On note en particulier une interaction antagoniste entre l'effet de la fertilisation et la forte compétition intracépée, ainsi qu'une tendance au retour aux fertilités relatives initiales dès 4-6 ans après l'application des traitements. Des recommandations sont néanmoins fournies pour obtenir des résultats optimums.
\end{abstract}

tallis / croissance / amélioration sylvicole / Betula pubescens Ehrh / Robinia pseudacacia L

Summary - Sylvicultural improvement experiments on the growth and regeneration of birch and Black locust tree in a traditional coppice. Preliminary results. Two experiments were carried out on 2 simple coppice stands (table l) located in Sologne and Orléanais (Central France) to increase growth and to improve regeneration after clear-cutting. Techniques for increasing the rootstock and stimulating growth were as follows : establishing plantations between stumps, increasing the sowing density of birch (Betula pubescens Ehrb) and the suckering of Black locust tree (Robinia pseudacacia $L$ ), the addition of fertilizer and ploughing (table (I).

The initial results are limited, showing: a negative effect for ploughing chiefly on the height growth of birch sprouts (fig 1), an antagonist interaction between the influence of fertilization and high sprout competition (fig 1), a short-lived stimulation on sowing installation (fig 4 and table V) and on suckering (fig 3). These results are probably due to very poor soils, intensive juvenile competition, and old root systems. A return to the initial fertility classification was observed 4-6 yr after treatment (table IV). Recommendations are given for obtaining optimum results.

coppice / growth / sylvicultural improvement / Betula pubescens Ehrh / Robinia pseudacacia $L$

* Correspondance et tirés à part. Adresse actuelle : INRA, station de recherches forestières, domaine de l'Hermitage, Pierroton, 33610 Cestas, France

** Adresse actuelle : INRA, station d'agronomie, domaine Saint-Paul, BP 91, 84140 Montfavet, France 


\section{INTRODUCTION}

Les taillis simples sont des peuplements forestiers se régénérant presque exclusivement par rejets de souche ou par drageons. lls représentent environ $20 \%$ de la surface productive de la forêt française. Ils sont caractérisés par une croissance juvénile annuelle en biomasse totale très forte culminant très précocement, par exemple dès 3-5 ans chez le châtaignier (Castanea sativa $L$ ) et le robinier (Pagès, 1986; Bédéneau, 1988), puis déclinant rapidement ensuite. Cette capacité juvénile de croissance est à mettre en parallèle avec l'existence d'un système racinaire quasi permanent et d'une souche riche en réserves nutritives sur laquelle se trouvent de nombreux bourgeons dormants.

Malgré ces potentialités a priori positives, ces peuplements montrent actuellement, en France, une croissance et une production relativement faibles, liées surtout à leur localisation et à leur mode de gestion. Différents auteurs ont relevé les facteurs défavorables à la croissance et à la régénération des taillis : ensouchement insuffisant et(ou) trop vieux, rotations trop longues, sols médiocres, espèces peu performantes et, dans une moindre mesure, modalités de coupe non-optimales (Mathey, 1931; Pardé, 1979; Riedacker, 1979; Blake et Raitanen, 1981; Riedacker et al, 1985). Le vieillissement des souches recouvre un ensemble de phénomènes liés à l'âge de l'ensouchement et aux modalités de coupe : remplacement des racines mères par des racines adventives à chaque recépage (Kazarjan, 1969), perte d'efficacité des bourgeons dormants (forte multiplication, tendance au regroupement, plus faible durée de vie : Kauppi et al, 1988), accumulation d'inhibiteurs phénoliques de la croissance (Blake et Raitanen, 1981). Il en résulte une augmentation de la mortalité des souches, et une diminution de la croissance et du nombre de rejets. En ce qui concerne la durée de rotation, elle conditionne la production moyenne obtenue en fin de rotation, selon des lois de croissance propres au taillis, avec des maximas pouvant culminer dès 10 ans chez le robinier (Pagès, 1986) et le châtaignier (Bédéneau, 1988). Elle conditionne également les possibilités d'installation des semis, aptes à renouveler l'ensouchement. D'autre part, l'espèce joue un grand rôle par sa capacité à rejeter, à drageonner et à fructifier (Parade, 1860; Mathey, 1931). Enfin, la fertilité du sol semble bien influer sur la production des taillis au niveau régional (Sabatier-Tarrago, 1989; Pagès, 1986; de Champs, 1972), mais selon des modalités qui restent à définir au niveau national pour ce traitement forestier (Bédéneau, 1988).

Différentes méthodes sylvicoles d'amélioration des taillis ont été expérimentées, mais ces essais sont peu nombreux. $\mathrm{Si}$ l'on excepte les transformations et les conversions en futaie, qui sortent du cadre de cette étude, les essais les plus fréquents concernent l'influence de la fertilisation du sol sur la production (Dubois, 1977; Mroz et al, 1985; Evans, 1986). Le rôle de la saison de coupe est surtout utilisé pour éliminer le taillis (Wenger, 1953). L'effet de la durée de rotation, souvent étudié en relation avec la densité de plantation dans les taillis à courte rotation, est, par contre, peu connu chez les taillis à vieil ensouchement (Riedacker et al, 1985; RullierBréval, 1985). Bien que de nombreux auteurs mentionnent la nécessité de renouveler l'ensouchement des taillis, par exemple par plantation ou semis (Parade, 1860; Mathey, 1931), et que ces pratiques puissent encore exister de nos jours (marcottage : Evans, 1984), elles ne semblent pas avoir été scientifiquement expérimentées. Il existe par contre des essais de dé- 
pressage visant à augmenter la production par diminution de la compétition juvénile (Dubois, 1977; Hocker, 1982).

Les essais que nous présentons ici concernent plus précisément l'étude expérimentale des possibilités d'augmentation de la croissance et de la production ainsi que d'amélioration de la régénération des taillis traditionnels, en demeurant dans le cadre du régime forestier du taillis; ils excluent donc les préocupations d'amélioration spécifique de la qualité de la production, comme le balivage (Hubert, 1983) ou la transformation directe en futaie (Lanier, 1986). lis ont été construits de manière à agir sur les facteurs de la croissance et les mécanismes de fonctionnement du taillis jugés majeurs à la lumière des connaissances actuelles. Le contexte favorable aux recherches sur les sources d'énergie renouvelable des années 1979-1985 (Chartier, 1987) a permis d'en assurer la promotion du fait de l'enjeu que représen- taient alors ces peuplements dans les ressources bois-énergie (Pardé, 1979; Riedacker, 1979). Ce contexte nous a amenés à retenir des solutions sylvicoles simples et peu coûteuses compatibles avec les limites économiques de la propriété privée où se trouve la presque totalité des taillis simples.

\section{MATÉRIELS ET MÉTHODES}

\section{Matériel végétal}

Nous nous sommes intéressés à 2 types de taillis simples couramment répandus en région Centre : un taillis de bouleau de Sologne (dispositif 1) et un taillis de robinier de l'Orléanais (dispositif 2). Les caractéristiques initiales de ces peuplements sont indiquées dans le tableau I. Les interventions ont porté sur la repousse du taillis après coupe à blanc du peuplement initial.

Tableau I. Principales caractéristiques des peuplements de taillis avant l'installation des dispositifs.

\begin{tabular}{lll}
\hline & \multicolumn{1}{c}{ Dispositifs } & \multicolumn{1}{c}{2} \\
\hline Localisation & La Ferté-St Aubin (Loiret) & Saint Denis-de-l'Hôtel (Loiret) \\
Sol & $\begin{array}{l}\text { Podzolique sablo-limoneux } \\
\text { à hydromorphie partielle }\end{array}$ & $\begin{array}{l}\text { Brun acide et } \\
\text { profond, sableux }\end{array}$ \\
Espèce dominante & Betula pubescens & Robinia pseudoacacia \\
Age (ans) & $30-35$ & 8 et 21 \\
Nombre de tiges/ha & 1600 à 3600 & 6600 et 6 000 \\
Hauteur dominante $\left(^{*}\right)(\mathrm{m})$ & 12,0 à 18,5 & 11,0 et 16,7 \\
Biomasse sur pied $(\mathrm{t} / \mathrm{ha})$ & 47 à 73 & 31 et 84 \\
\hline
\end{tabular}

(") Moyenne des hauteurs des 100 plus gros arbres à l'ha. 


\section{Dispositifs}

Les types de techniques utilisées : plantation intercalaire, fertilisation/amendement et travail du sol, décrits ci-dessous, ont été appliqués séparément dans les 2 dispositifs installés, selon diverses modalités, comme indiqué dans le tableau II. Les répétitions sont organisées en blocs agronomiques complets non-éclatés, à raison de 2 blocs (dispositif 1) ou de un seul bloc (dispositif 2) par parcelle forestière homogène. Dans le cas du dispositif $n^{\circ} 1$, les placeaux unitaires sont séparés par des bandes neutralisées larges de $10 \mathrm{~m}$. La croissance a été suivie durant 2 ans (dispositif 2) à 6 ans (dispositif 1) après le recépage initial. Du fait de l'exploitation tardive (août 82) de la moitié Nord du dispositif 1 , on y a considéré l'âge de la repousse inférieure de 1 an à celui de la moitié Sud, et les traitements y ont été effectués 1 an plus tard.

\section{Techniques syivicoles}

Trois techniques différentes d'augmentation de la densité des peuplements, adaptées aux particularités des espèces, ont été expérimentées. Tout d'abord, l'installation de plants entre les souches après le recépage (dispositif 1) a eu pour objectif d'obtenir une densité globale (cépées + plants destinés à être recépés) optimale de l'ordre de 2500 à 3500 individus/ha, densité observée dans les meilleurs taillis de moins de 20 ans (Mathey, 1931). Nous avons ainsi installé des plançons d'un même clône de Populus trichocarpa, var Fritzy Pauley à raison de 1 000/ ha, l'année de la repousse du taillis; ils ont été complétés 2 ans plus tard par des plants d'une même provenance de Quercus rubra $L$, à raison de $1000 / \mathrm{ha}$; les plants ont bénéficié d'une protection vis-à-vis du gibier dès la plantation : gaine spirale plastique autour des plançons de peuplier (Populus trichocarpa Fritzy Pauley), ré-

Tableau II. Principales caractéristiques des dispositifs installés et répartition des traitements sylvicoles utilisés.

\section{Dispositifs}

1

Début 1982

11200

4

700

Travail superficiel

du sol au rouleau landais

Plantation intercalaire de peupliers trichocarpa et de chénes rouges

Amendement-fertilisation en phosphate naturel et en nitrate potassique

Témoin
Début 1983

1600

2

200
Modalités 
pulsifs puis fourreaux grillagés ( $1 \mathrm{~m}$ de diamètre et $1,50 \mathrm{~m}$ de hauteur) autour des chênes rouges (Quercus rubra L). Leur croissance a également été favorisée par recépage des plançons au stade 1 an dans les blocs 3 et 4 et apport d'engrais retard : $10 \mathrm{~g} / \mathrm{plant}$ de N-P-K (7-14-10) au pied des chênes rouges. D'autre part, le travail superficiel du sol au rouleau landais de $1,1 \mathrm{t}$ (dispositif 1) a visé à favoriser l'installation et la survie de semis (bouleau). Enfin, la stimulation du drageonnement (dispositif 2) a été tentée d'une part en travaillant le sol entre les souches à $10-30 \mathrm{~cm}$ de profondeur, soit uniformément (traitement labour) soit seulement sur une couronne à $30 \mathrm{~cm}$ autour des souches (traitement pioche) de façon à induire un drageonnement du robinier par blessure des racines (Vaulot, 1914; Mattoon, 1930); et d'autre part en recouvrant les étocs à l'aide d'une bâche plastique verte, afin de limiter le développement des rejets de souche.

L'amélioration des caractéristiques du sol du dispositif 1 a été réalisée par ailleurs à l'aide d'un apport de phosphate calcique permettant a la fois de remonter le $\mathrm{pH}$ et d'augmenter la quantité de phosphore, caractéristiques presque toujours limitantes dans les sols forestiers (Duchaufour, 1970), et peu coûteuses à améliorer. Un apport de nitrate potassique a également été effectué à titre de complément. Les doses et époques d'épandage ont été les suivantes :

200 U/ha $\mathrm{P}_{2} \mathrm{O}_{5}$ fin avril 1982 (Sud) ou 1983 (Nord)

$46 \mathrm{U} / \mathrm{ha} \mathrm{N}+164 \mathrm{U} / \mathrm{ha} \mathrm{K}_{2} \mathrm{O}$ en mars 1983 (Sud ou 1984 (Nord)

\section{Mesures}

Le suivi de la croissance et de la production de la jeune repousse de bouleau a été effectué selon la méthode décrite par Cabanettes (1989) : sur chaque placeau unitaire, mesure du nombre de rejets et de la hauteur du plus grand rejet (ou maître-rejet) de chaque cépée, mesure du diamètre et de la longueur de tous les rejets d'une dizaine de cépées échantillons, et prélèvement destructif d'échantillons sur zone de protection périphérique permettant d'établir des ta- rifs biomasse au niveau rejet. Pour le taillis de robinier, compte tenu du drageonnement, la totalité des tiges a dû être mesurée (Pagès, 1986).

Les prélèvements foliaires, destinés à contrôler l'absorption des éléments minéraux apportés dans le dispositif $\pi^{\circ} 1$, ont été effectués en 1985 selon le protocole recommandé par Bonneau (1988) : échantillonnage effectué fin août sur une dizaine de cépées de croissance moyenne tirées au hasard pour chaque placeau; les feuilles ont été recueillies dans le tiers supérieur de rejets dominants ou codominants. Les analyses minérales (éléments majeurs) ont été réalisées par le laboratoire agréé Sicagro sur échantillons de feuilles broyées et séchées à $65^{\circ} \mathrm{C}$.

\section{Analyse des données}

La comparaison statistique des traitements a été effectuée par analyse de variance ou analyse de régression. Du fait de la non-normalité vérifiée des variables, nous avons utilisé l'analyse de variance non-paramétrique de Friedman (Dagnélie, 1970); la comparaison individuelle d'un traitement au témoin a été effectuée à laide d'un test unilatéral non-paramétrique (Dacunha-Castelle et Tomassone, 1975). L'analyse de régression a été réalisée lorsqu'une covariable $X$ utile a pu être identifiée : on a alors réduit la variabilité indépendante des traitements en analysant l'effet de ces derniers sur la valeur des paramètres des régressions reliant la variable principale $Y$ à $X$. Ceci a pu être réalisé pour les relations entre nombres de rejets par cépée à des âges différents, ainsi qu'entre la longueur et le diamètre des rejets à un âge donné. Le modèle de régression non-linéaire retenu est du type :

$$
Y=a X^{B}+b
$$

Nous avons utilisé le logiciel Genstat (Astier et al, 1982) pour le paramétrage du modèle (1) selon les traitements, le test $F$ (Snedecor et Cochran, 1957) pour la comparaison globale des modèles et sous-modèles, et le test $t$ de Student corrigé pour la comparaison individuelle des pentes. 


\section{RÉSULTATS}

\section{Effets de lamendement-fertilisation (dispositif $n^{\circ}$ 1)}

\section{Effets sur la croissance}

Nous constatons surtout une influence de la fertilisation sur le nombre de rejets de Bouleau pubescent par cépée : la figure 1 montre une tendance à un retard de mortalité des rejets entre 2 et 4 ans sur les placeaux fertilisés (différences non statistiquement significatives au seuil de $5 \%$ ), et surtout un accroissement de cette mortalité entre 4 et 6 ans. Ce dernier résultat est confirmé par l'analyse de la régression du nombre des rejets par cépée à 6 ans sur celui des rejets à 4 ans : la différence globalement significative est vérifiée dans 3 blocs sur 4 , et ne dépend donc pas du nombre initial de rejets à 4 ans. Pour la croissance en diamètre et en longueur des rejets, l'analyse est délicate car les cé- pées-échantillons choisies diffèrent d'un inventaire à l'autre. Si globalement les placeaux plantés et fertilisés ont les diamètres et les longueurs significativement les plus élevés, il existe une forte interaction avec le bloc (c'est le cas pour 2 blocs sur 4) et surtout avec les diamètres moyens du peuplement initial. En revanche, la pente de la régression de la longueur des rejets sur leur diamètre est significativement supérieure à 2 ans dans les placeaux fertilisés (c'est le cas dans les 4 blocs); mais cette différence finit par s'annuler à 6 ans (tableau III). Au total, la fertilisation n'a pas augmenté significativement la production globale, et nous assistons plutôt à un retour progressif au classement initial des fertilités, comme en témoigne le tableau IV pour plusieurs accroissements moyens annuels (évolution du coefficient de corrélation vers des valeurs de plus en plus positives); la figure 5 montre en particulier une tendance à un retour au classement initial des allongements moyens des rejets.

\section{NOMBRE DE REJETS / CEPEE}
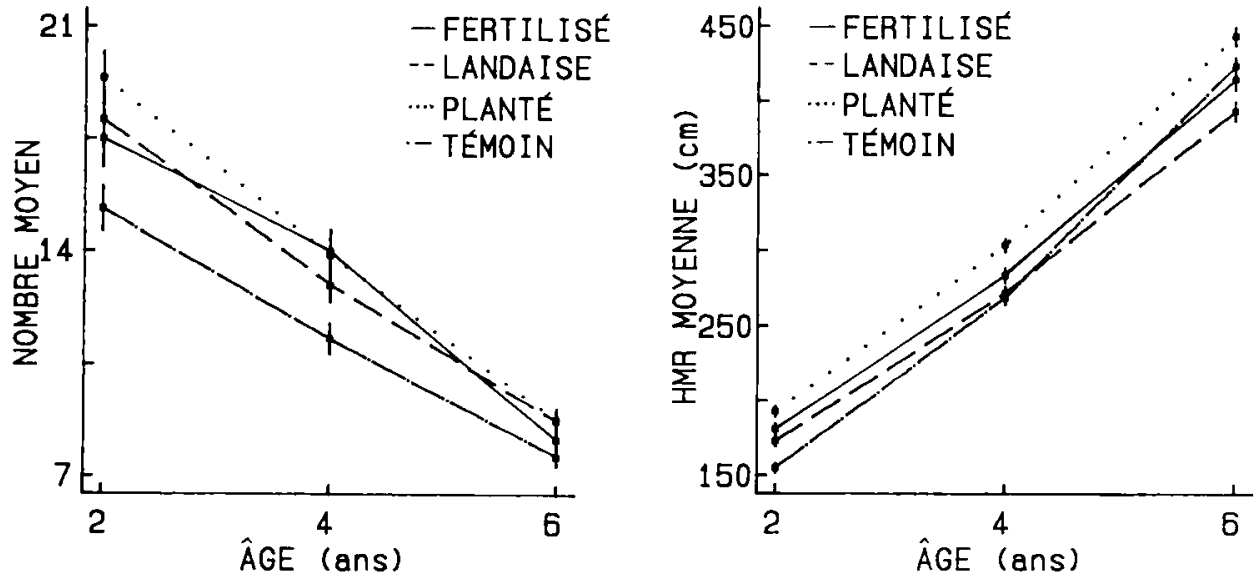

Fig 1. Évolution de 2 variables de croissance entre 2 et 6 ans après recépage selon le traitement appliqué. Cas du dispositif $n^{\circ} 1$ (bouleau pubescent). Chaque point correspond à la moyenne des valeurs obtenues pour les 4 blocs, et les barres verticales représentent les écarts types correspondants. 
Tableau III. Évolution des pentes des régressions reliant la longueur au diamètre des rejets de bouleau pubescent (modèle non-linéaire (1)) selon l'âge des rejets (dispositif $n^{\circ} 1$ ). Les pentes sont exprimées en $\mathrm{cm} / \mathrm{mm}$. À l'intérieur de chaque bloc, seules les valeurs suivies d'une lettre différente diffèrent entre elles au seuil de $5 \%$.

\begin{tabular}{llrl}
\hline & \multicolumn{2}{c}{ Age } \\
\multicolumn{2}{c}{ Bloc traitement } & 2 ans & 6 ans \\
\hline & & & \\
\hline & Fertilisé & $7,90^{\mathrm{a}}$ & $24,7^{\mathrm{a}}$ \\
& Rouleau landais & $7,24^{\mathrm{b}}$ & $24,6^{\mathrm{a}}$ \\
& Planté & $8,37^{\mathrm{a}}$ & $23,0^{\mathrm{b}}$ \\
& Témoin & $7,15^{\mathrm{b}}$ & $23,0^{\mathrm{b}}$ \\
& Fertilisé & $8,80^{\mathrm{a}}$ & $25,6^{\mathrm{ab}}$ \\
& Rouleau landais & $7,75^{\mathrm{b}}$ & $24,6^{\mathrm{a}}$ \\
& Planté & $7,53^{\mathrm{b}}$ & $26,9^{\mathrm{b}}$ \\
& Témoin & $7,92^{\mathrm{b}}$ & $26,7^{\mathrm{b}}$ \\
& Fertilisé & $10,06^{\mathrm{a}}$ & $25,1^{\mathrm{a}}$ \\
& Rouleau landais & $7,36^{\mathrm{b}}$ & $25,2^{\mathrm{ab}}$ \\
& Planté & $7,70^{\mathrm{b}}$ & $25,8^{\mathrm{ac}}$ \\
& Témoin & $9,33^{\mathrm{c}}$ & $26,7^{\mathrm{bc}}$ \\
& Fertilisé & $10,06^{\mathrm{a}}$ & $23,8^{\mathrm{a}}$ \\
& Rouleau landais & $7,36^{\mathrm{b}}$ & $23,7^{\mathrm{a}}$ \\
& Planté & $6,25^{\mathrm{c}}$ & $26,1^{\mathrm{b}}$ \\
& Témoin & $7,27^{\mathrm{b}}$ & $26,9^{\mathrm{b}}$ \\
& & & \\
\hline
\end{tabular}

\section{Analyses foliaires (fig 2)}

Les teneurs en éléments majeurs du bouleau pubescent sont situées dans les fourchettes optimales fournies pour les feuillus peu exigeants par Bonneau (1988) et sont comparables aux valeurs citées par Ferm et Markkola (1985) pour la même espèce au même âge traitée en taillis. Suite à l'apport de $\mathrm{P}$ et $\mathrm{Ca}$ puis $\mathrm{N}$ et $\mathrm{K}$, nous constatons qu'après 1-3 ans, seule la concentration en potassium est supérieure dans les placeaux fertilisés par rapport à celle des placeaux témoins, de $28 \%$ en moyenne. Pour le phosphore et l'azote, ce n'est le cas que sur une moitié du dispositif (diffé- rente pour $P$ et pour $N$ ), la concentration en calcium étant relativement constante.

\section{Effets du travail du sol (dispositifs $n^{\circ} 1$ et 2)}

\section{Effets sur la croissance du taillis}

Nous voyons sur la figure 1 que le travail du sol dans le dispositif $n^{\circ} 1$ a nettement déprimé la croissance en hauteur des maîtres-rejets entre 2 et 6 ans, cet effet tendant à s'accroître au cours du temps (la diminution de l'accroissement passe de $37 \%$ à $56 \%$ ). Bien que l'influence de ce traitement soit globalement significative sur cette variable entre 2 et 6 ans, elle est surtout nette dans les blocs 3 et 4 où le rouleau landais est passé sur les souches (pour les blocs 1 et 2, le sol n'a été travaillé qu'entre les souches). D'autre part, le rapport par placeau du nombre de cépées vivantes de bouleau après la coupe au nombre de tiges de bouleau du peuplement initial est minimal dans les blocs 3 et 4 pour les placeaux travaillés, et inférieur de $20 \%$ à $23 \%$ à celui du placeau témoin. Le regroupement des tiges initiales en cépées pouvant être considéré comme indépendant du placeau d'un même bloc, ce résultat tend à montrer un effet du travail du sol sur la mortalité des souches de bouleau. Au total, à 6 ans, la production en biomasse de bouleau des placeaux travaillés est inférieure à celle des placeaux témoins; cette différence est significative à $5 \%$ pour les blocs 3 et 4 .

Dans le dispositif $n^{\circ} 2$, les traitements pioche et surtout labour ont provoqué une forte augmentation du nombre de rejets dès la première année (+ $43 \%$ à $99 \%$ respectivement par rapport au témoin). Ce phénomène tend ensuite à disparaître au cours du temps (- $8 \%$ à $+20 \%$ à 4 ans). En termes de production en biomasse, la 

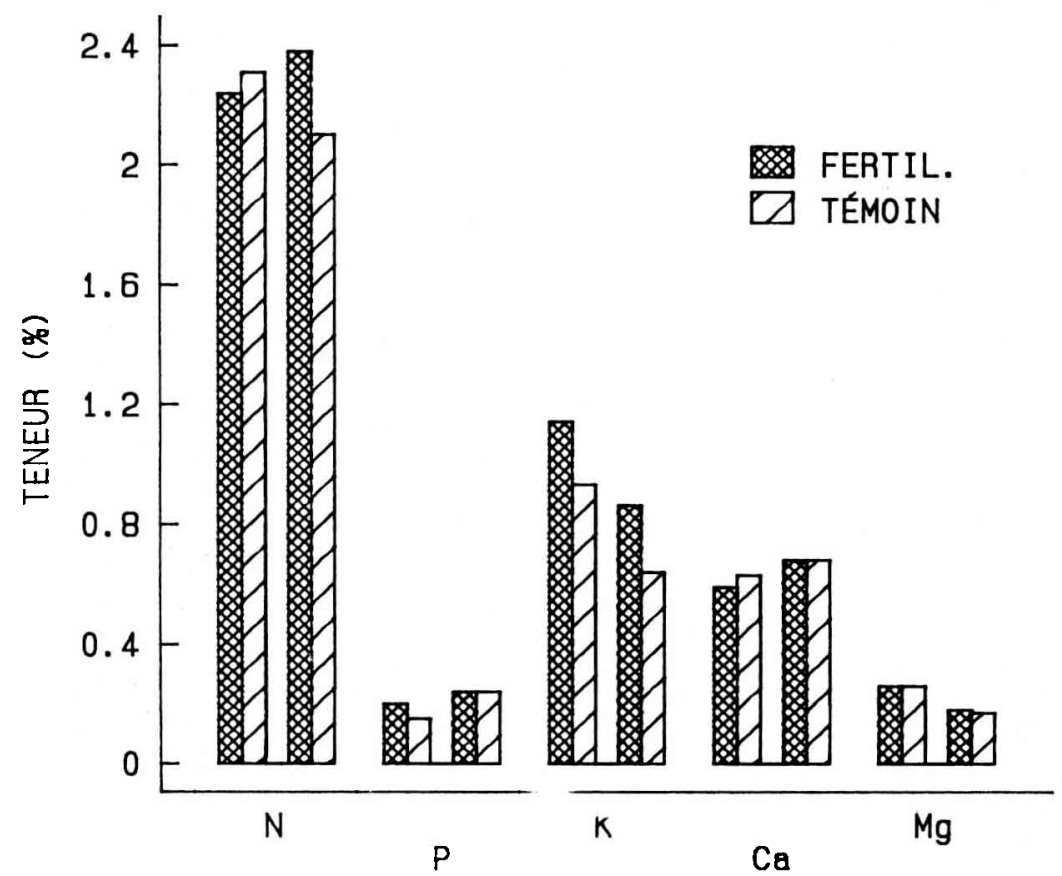

Fig 2. Résultats des analyses minérales foliaires effectuées dans les placeaux témoins et fertilisés du dispositif $n^{\circ} 1$ (bouleau pubescent), sur des rejets âgés de 3-4 ans. Pour chaque élément minéral, les valeurs représentées à gauche correspondent aux moyennes des blocs 1 et 2 , celles de droite aux moyennes des blocs 3 et 4 .

stimulation initiale est comparable (+ $63 \%$ à $+92 \%$ ), mais elle se maintient un peu mieux pour le traitement labour $(+40 \%$ à 4 ans). La modalité bâche a un effet plutôt négatif sur la production. Pour le bloc 1 , où le peuplement initial était le plus jeune (8 ans) et la densité initiale plus élevée (6 600 tiges/ha contre 6000 tiges/ha en bloc 2), la stimulation du drageonnement, tant en nombre de rejets qu'en production, a été plus importante que dans le bloc 2 , surtout pour la modalité labour. A 4 ans, le bloc 1 se caractérise par le maintien d'une forte production de drageons, liée surtout à leurs dimensions, dans le traitement labour.

\section{Effets sur l'installation des semis (dispositif $n^{\circ} 1$ )}

L'estimation et le suivi de la quantité de semis ont fait l'objet d'une notation semiquantitative à 5 niveaux (absence, présence, et 3 niveaux d'abondance croissante) effectuée par carrés de $3 \mathrm{~m} \times 3 \mathrm{~m}$ sur la totalité de la surface de chaque placeau durant les étés 1983 et 1985 . Les résultats concernant le taux de présence (rapport du nombre de carrés où il y a des semis au nombre total de carrés) sont mentionnés sur la figure 4 . Le tableau $\mathrm{V}$ montre l'évolution comparée du pourcentage de carrés où les semis de bouleau 

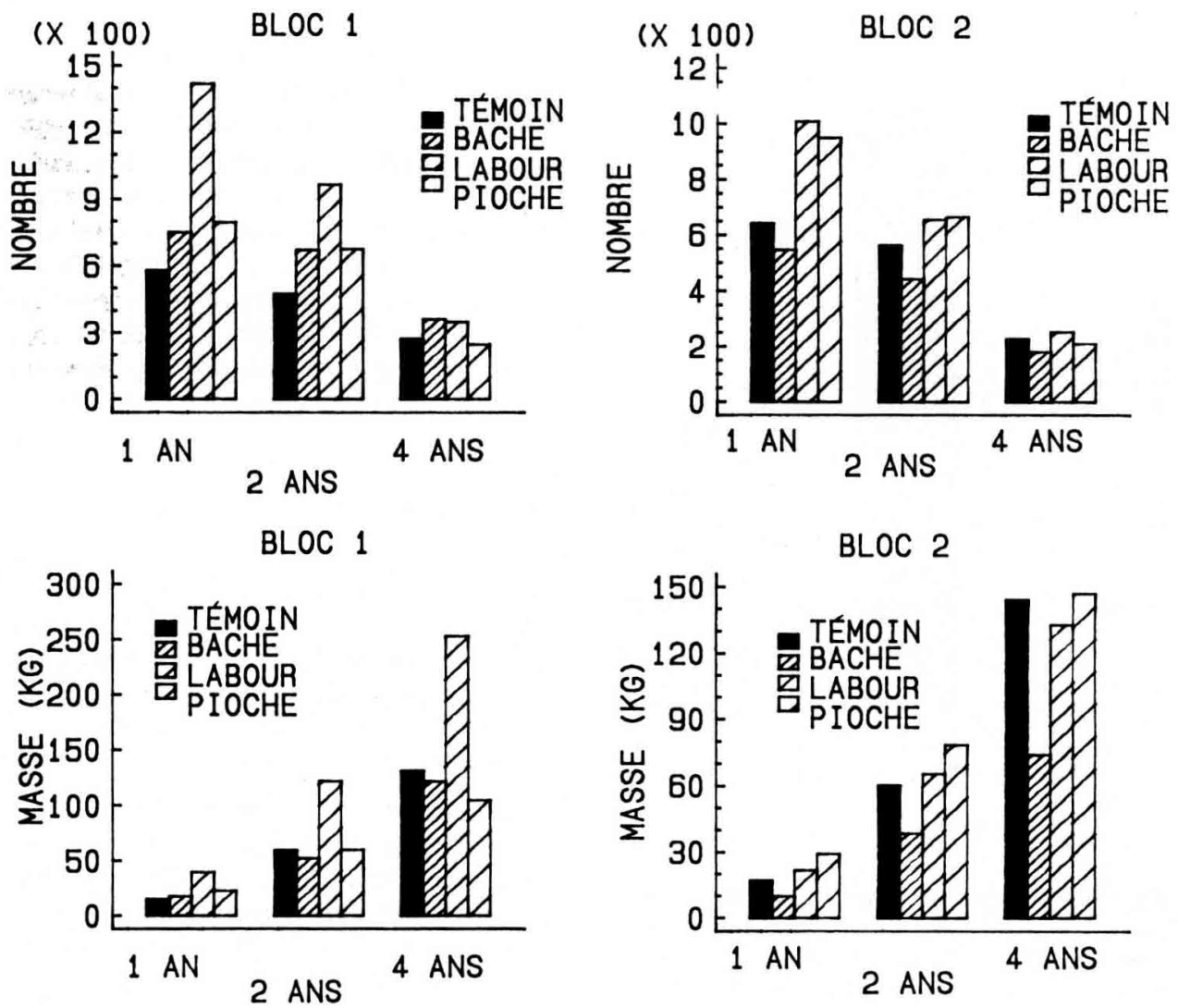

Fig 3. Évolution de la repousse du taillis de robinier (dispositif $n^{\circ} 2$ ), exprimée en nombre de rejets et en biomasse totale, $1-4$ ans après recépage et application des traitements. Les valeurs correspondent à des surfaces unitaires de $156 \mathrm{~m}^{2}$.

sont abondants ou seulement présents. En 1983, le taux de présence est plus élevé sur les placeaux travaillés $(+10 \%)$; pour le bouleau, la différence se situe essentiellement au niveau du nombre de carrés où les semis sont présents mais nonabondants, ce qui indique davantage un effet du travail du sol sur une meilleure répartition des semis que sur leur quantité. Entre 1983 et 1985 , il y a une forte diminution de la présence des semis $(-45 \%)$ qui concerne davantage les placeaux travaillés et, dans ceux-ci, plus particulière- ment le pourcentage de carrés où les semis de bouleau sont présents mais nonabondants $(-30 \%)$. Ce phénomène montre que ce sont surtout les semis de bouleau relativement isolés qui disparaissent, particulièrement ceux qui avaient pu s'installer initialement grâce au travail du sol. Les observations de terrain précisent en outre que l'installation et la survie des semis sont très favorisées par l'humidité locale du sol, caractéristique du microsite et/ou corrélée avec le micro-relief préexistant (billonnage); la survie est par contre très 


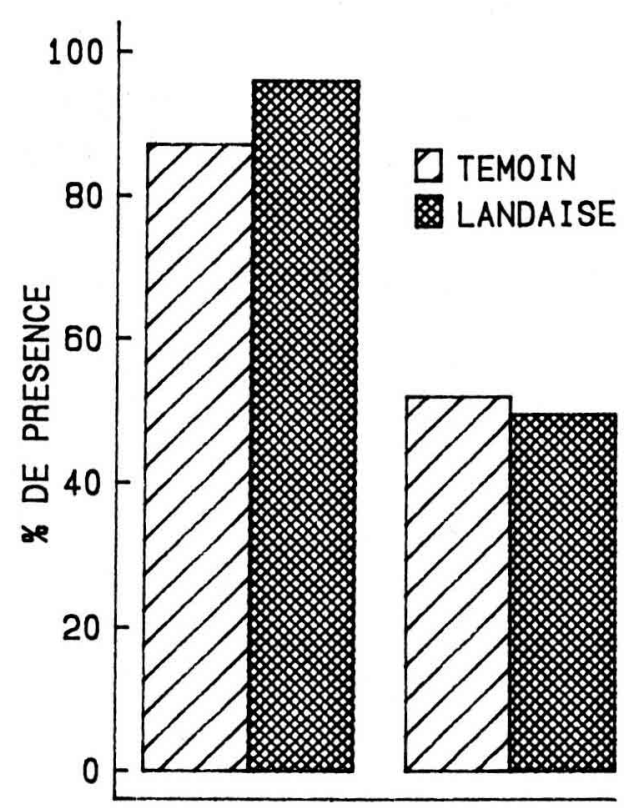

1983

1985

Fig 4. Installation comparée des semis selon le traitement aux stades 2 ans et 4 ans après passage du rouleau landais (dispositif $n^{\circ} 1$ ). Le taux de présence correspond à la moyenne du rapport du nombre de mailles où existent des semis au nombre total de mailles d'un placeau.

compromise partout où la végétation basse (graminées, bruyère ou fougère) reprend avec vigueur.

\section{Plantations intercalaires (dispositif $n^{\circ}$ 1)}

La forte mortalité des peupliers a conduit à effectuer leur recépage partiel (blocs 3 et 4) et une plantation complémentaire de chênes rouges. Après 5 ans d'installation, nous avons noté globalement plus de $90 \%$ de peupliers morts, sauf dans le bloc 2
(33\%). Pour les chênes rouges, la mortalité totale a été comprise entre 20 et $30 \%$ durant les 4 premières années d'installation à l'exception du bloc $2: 1 \%$ ), et le nombre de nouveaux plants morts tend actuellement à devenir très faible. Toutefois, dans les blocs 3 et 4 , où l'ensouchement est plus dense, un dépressage des cépées de bouleau a été nécessaire à 6 ans pour éviter une trop forte concurrence (hauteur des rejets : $4 \mathrm{~m}$; hauteur des plants : $1 \mathrm{~m}$ ).

\section{DISCUSSION}

Les 2 essais installés ont livré une première série de résultats mettant en évidence l'influence ou l'absence d'influence des techniques expérimentées sur les paramètres de la croissance et de la régénération de taillis traditionnels.

Les apports minéraux ont été diversement retrouvés au niveau foliaire 1 à 3 ans après leur introduction. Ces résultats peuvent être attribués au comportement particulier de l'espèce et (ou) à des phénomènes de limitation de la part du milieu, comme l'ont déjà observé Mroz et al (1985) et Evans (1986). Le sol du taillis de bouleau était en effet particulièrement pauvre et peu profond. Sa teneur en potassium $(0,05$ meq pour $100 \mathrm{~g})$ était proche du seuil de carence, ce qui pourrait expliquer l'absorption nette et générale de cet élément dans ce dispositif, et également sa nécessité. Les différences constatées entre blocs peuvent être liées aux années différentes de fertilisation. Mais l'apport d'azote semble être plus efficace dans les blocs 3 et 4 du dispositif 1 , où la quantité d'azote du sol est davantage limitante $(0,15 \%$ o contre $0,5 \%$ o dans les blocs 1 et 2$)$.

Le faible effet global de lamendementfertilisation pourrait être dû à un rapport N/ $K$ des apports légèrement insuffisant. L'effet le plus net est celui qui concerne le 
Tableau IV. Comparaison des classements des placeaux du dispositif $n^{\circ} 1$ avant et après l'application des traitements. La comparaison porte sur les accroissements moyens annuels des variables indiquées, qui sont confrontés aux valeurs initiales à l'aide du coefficient de corrélation de rang de Spearman.

\begin{tabular}{lccc}
\hline \multicolumn{1}{c}{ Variables comparées } & \multicolumn{3}{c}{ Stade après coupe } \\
& 2 ans & 4 ans & 6 ans \\
\hline $\begin{array}{l}\text { Hauteur dominante initiale } \\
\text { et hauteur moyenne } \\
\text { des maîtres-rejets }\end{array}$ & $-0,75$ & $+0,60$ & $+0,73$ \\
$\begin{array}{l}\text { Moyenne des circonferences initiales } \\
\text { à } 1,30 \text { m et moyennes des diamètres }\end{array}$ & $+0,40$ & $+0,45$ & $+0,61$ \\
$\begin{array}{l}\text { Production initiale de bouleau } \\
\text { et production de la repousse }\end{array}$ & $-0,52$ & $-0,24$ & $+0,16$ \\
\hline
\end{tabular}

nombre de rejets par cépée de Bouleau. ॥ appelle plusieurs remarques. Tout d'abord, ce phénomène n'a pas été observé sur d'autres espèces dans des conditions expérimentales voisines (Mroz et al, 1985). Les 2 phases successives de ralentissement puis d'accélération de la mortalité peuvent être mises en parallèle avec les 2 phases d'apports minéraux, décalées d'1 an ( $\mathrm{P}-\mathrm{Ca}$ puis $\mathrm{N}-\mathrm{K})$. II peut également s'agir de l'expression de mécanismes physiologiques liés à l'absorption minérale, à la structure et au fonctionnement des souches et de leur appareil racinaire : phénomène d'épuisement de l'apport minéral, et (ou) d'intensification de la compétition

Tableau V. Pourcentage de carrés de $3 \times 3 \mathrm{~m}$ où les semis de bouleau sont abondants $(A)$ ou seulement présents $(P)$ dans les placeaux travaillés au rouleau landais et dans les placeaux témoins du dispositif $n^{\circ} 1$ entre 1983 et 1985.

\begin{tabular}{|c|c|c|c|c|c|c|c|c|}
\hline & \multicolumn{4}{|c|}{1983} & \multicolumn{4}{|c|}{1985} \\
\hline & \multicolumn{2}{|c|}{ Témoin } & \multicolumn{2}{|c|}{ Landaise } & \multicolumn{2}{|c|}{ Témoin } & \multicolumn{2}{|c|}{ Landaise } \\
\hline & $P$ & $A$ & $P$ & $A$ & $P$ & $A$ & $P$ & $A$ \\
\hline Bloc 1 & 46 & 14 & 45 & 44 & 1 & 14 & 50 & 6 \\
\hline Bloc 2 & 35 & 62 & 70 & 15 & 44 & 26 & 12 & 22 \\
\hline Bloc 3 & 23 & 1 & 71 & 16 & 38 & 20 & 31 & 10 \\
\hline Bloc 4 & 43 & 7 & 46 & 49 & 5 & 9 & 21 & 23 \\
\hline Moyenne & 37 & 21 & 58 & 31 & 22 & 17 & 28 & 15 \\
\hline
\end{tabular}


entre les rejets d'une même cépée. En tout état de cause, il apparaît une interaction entre la fertilisation et la compétition entre rejets, cette interaction pouvant contribuer à masquer d'autres effets positifs de la fertilisation, notamment sur la croissance en diamètre et en hauteur des rejets comme l'ont observé d'autres auteurs (Czapowskyj et Safford, 1979; Herbert, 1983; Ferm et al, 1982). La diminution du rapport longueur/diamètre des rejets des placeaux fertilisés au cours du temps traduit peut-être un effet de la fertilisation sur la forme des rejets, avec une stimulation plus importante de l'accroissement en diamètre. Herbert (1983) a observé un phénomène semblable sur eucalyptus, principalement sous l'effet d'un apport d'azote. Mais ce phénomène pourrait aussi être lié à l'évolution de la mortalité, les très petits rejets qui tendent à disparaître étant caractérisés par un rapport longueur/diamètre plus élevé.

La reconstitution du classement des vigueurs initiales 4-6 ans après le recépage initial s'observe particulièrement pour le classement des blocs (fig 5), mais également pour celui des placeaux à l'intérieur de chaque bloc pour 3 blocs sur 4 . II s'agit très certainement là d'une influence déterminante des variations du milieu, ou du génotype, qui a participé à réduire l'effet et la durée des traitements appliqués.

L'augmentation de la mortalité des souches de bouleau sous l'effet du rouleau landais s'explique par le traumatisme important causé sur la surface aérienne des souches, avec arrachement de l'écorce, susceptible d'éliminer les bourgeons dor-

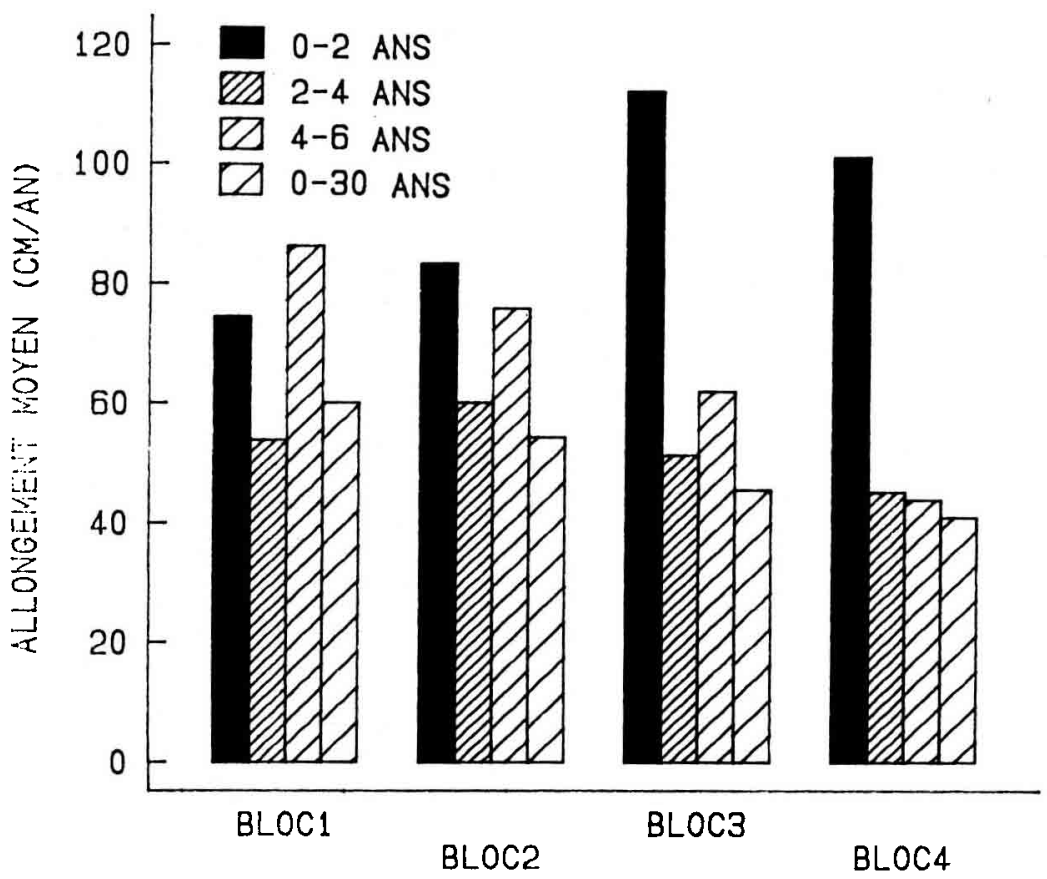

Fig 5. Allongement moyen annuel des rejets de bouleau pubescent selon l'âge et le bloc. Les chiffres correspondent aux moyennes de l'allongement des maitres-rejets des cépées pour la repousse (0-6 ans), et aux moyennes de l'allongement des arbres dominants pour le peuplement initial (0-30 ans). 
mants. Ce résultat est à relativiser, compte tenu de la fragilité des souches de bouleau, peu «longévives». En ce qui concerne la limitation de la croissance en hauteur des cépées, nous pouvons penser à un traumatisme de l'appareil racinaire provoqué par le rouleau landais : chez une espèce dont le renouvellement des racines est peu marqué (Bédéneau et Pagès, 1984), ce traumatisme pourrait entraîner une diminution du volume de sol exploité. Les observations de terrain suggèrent par ailleurs l'intervention possible d'un effet indirect du rouleau landais : nous assistons à un envahissement progressif des placeaux travaillés par la bruyère (Erica cinerea, Calluna vulgaris), susceptible de concurrencer le bouleau vis-à-vis de l'eau du sol.

Pour l'installation des semis de bouleau entre les souches, il apparaît qu'un travail moins uniforme du sol est préférable, afin de favoriser le développement de plages de semis dans de bonnes conditions hydriques; il est nécessaire d'y associer, au moins dans ce type de milieu, un traitement herbicide sélectif permettant d'éliminer la compétition de la végétation basse.

L'effet du travail du sol sur le drageonnement du robinier montre qu'outre l'interaction avec la forte compétition juvénile, qui annule rapidement l'augmentation du nombre des drageons, nous pouvons obtenir une amélioration plus durable de leur croissance individuelle. Nous pouvons penser que les blessures racinaires, qui induisent la formation de racines adventives, ont amélioré l'alimentation des drageons par augmentation du nombre des racines actives et (ou) par réduction des distances tige-racine. Par ailleurs, les différences observées entre les intensités de réaction des peuplements des 2 blocs pourraient indiquer l'importance du rôle de l'âge du peuplement initial, et donc de son système racinaire. Le travail du sol autour des souches a donné de moins bons résultats dans l'ensemble, ce qui peut être interprété comme l'effet de la compétition interne à la couronne de drageons résultants ou de la concurrence des rejets de la cépée qu'ils entourent.

Pour les plantations intercalaires, le principal problème rencontré est dû à la sécheresse estivale : la forte mortalité des peupliers et la meilleure survie des plants et plançons dans le bloc 2, plus humide, en attestent nettement. L'ensouchement est suffisamment faible pour que les cépées ne concurrencent pas trop les plants; il assure même sans doute un abri climatique, compte tenu des faibles mortalités enregistrées.

\section{CONCLUSION}

Ce premier bilan de tentatives d'amélioration de la croissance et de la régénération de taillis traditionnels fournit dans l'ensemble des résultats soit négatifs (effet du passage d'un rouleau landais sur la croissance du bouleau) soit de portée limitée en intensité (amendement-fertilisation) ou en durée (installation des semis, drageonnement). Le rôle des plantations intercalaires n'a pu encore être quantitativement défini, le recépage des plants étant encore prématuré : les modalités sylvicoles à appliquer ultérieurement restent en effet à définir (âge du premier recépage des plants, en tenant compte des caractéristiques biologiques des espèces en présence). II mériterait d'être précisé par la poursuite des mesures, compte tenu des bonnes performances des plants de chêne rouge. Ces résultats seront complétés ultérieurement par ceux d'essais plus récemment installés (1984 et 1986) concernant l'étude de l'influence de la durée de rotation sur la croissance d'un taillis de châtaignier (Castanea sativa Mill), et la re- 
cherche de densités optimales de plants en plantation intercalaire dans un taillis de charme (Carpinus betulus L).

Les principales difficultés rencontrées ont été tout d'abord la grande variabilité initiale du milieu et des peuplements, entraînant l'existence de blocs demeurant encore très hétérogènes, malgré les précautions prises. Ensuite, les caractères défavorables à la croissance de la plupart des sols, tant en termes minéraux qu'hydriques, ont probablement constitué des facteurs limitant les réponses de croissance aux traitements expérimentés. Ainsi pour le dispositif $n^{\circ} 1$, nous assistons à un important déficit hydrique en été, lié au caractère très sableux et superficiel du sol; par ailleurs, la montée de la nappe phréatique en hiver favorise probablement l'enracinement à faible profondeur, et le régime du taillis accentue encore cet état de fait car les nouvelles racines y sont souvent latérales et superficielles. Nous avons observé d'autre part des phénomènes inattendus d'interaction entre l'enrichissement minéral du sol et la mortalité juvénile intense des rejets. Enfin, il n'a pas été possible techniquement de suivre individuellement les cépées au moment du recépage initial, ce qui a limité les comparaisons entre repousse et peuplement initial et n'a pas permis une ségrégation optimale de l'effet des traitements vis-à-vis de la variabilité initiale. Toutefois, nous avons pu observer dans le dispositif $n^{\circ} 1$ une tendance de la repousse à reconstituer les variations initiales 6 années après le recépage; cette observation confirme les hypothèses sur l'existence de facteurs limitants.

Ces premiers résultats permettent toutefois de dégager quelques recommandations pour de futurs essais de même nature. Le travail du sol avec passage d'engins sur les souches est à proscrire, sauf peut-être chez les espèces drageonnantes. Dans ce dernier cas, il semble pré- férable d'effectuer l'opération à partir d'un peuplement assez jeune, dont le système racinaire est plus réactif. Le bénéfice à attendre est de courte durée, et convient donc surtout pour des rotations de 3-5 ans par exemple. Les apports minéraux, même en quantités notoirement suffisantes (sauf peut-être pour l'azote), peuvent interagir avec la forte mortalité juvénile, tout au moins chez les espèces à grand nombre initial de rejets par cépée, avec l'efficacité de l'appareil racinaire, et avec le déficit hydrique ou la faible rétention du sol. II ne faut donc les conseiller qu'en cas de carence évidente (analyse foliaire) et sur des sols pas trop dégradés. Les problèmes hydriques rencontrés (sécheresse d'été, montée de la nappe phréatique l'hiver) tendraient à suggérer, particulièrement en Sologne, l'irrigation estivale et le drainage hivernal afin de permettre au système racinaire de descendre et d'augmenter ainsi la réserve utile à la souche; mais il s'agit là de techniques coûteuses sans rapport avec la valeur commerciale de ces taillis. Au total, la combinaison d'une technique d'augmentation de l'ensouchement (plantation ou semis) avec l'utilisation d'une durée de rotation optimale, donc relativement courte mais adaptée à l'espèce et au sol, semble constituer le meilleur moyen d'augmenter la croissance et la production totale, dans les limites économiques de la forêt privée française et dans le cadre du régime du taillis simple.

\section{REMERCIEMENTS}

Nous tenons à remercier vivement les équipes techniques de la station de sylviculture et de I'UEBF de I'INRA d'Orléans ainsi que l'Agence française pour la maîtrise de l'énergie, qui ont permis techniquement et financièrement l'installation et le suivi des essais. Les antennes locales des CRPF et de I'ONF, particulièrement MM Formery et Raoul, nous ont apporté une 
aide appréciée pour la recherche des peuplements. Nous sommes reconnaissants aux propriétaires privés, $M^{m e}$ de Fontbelle et $M$ Maes, d'avoir accepté l'instaliation de nos essais sur leur propriété.

\section{RÉFÉRENCES}

Astier R, Bouvier A, Coursol J, Denis JB, Dervin $C$, Jolivet $E$, Lesquoy $E$, Pons $O$, Tomassone R, Vila JP (1982) GENSTAT, un langage statistique. INRA Biométrie, Paris, $555 \mathrm{p}$

Bédéneau $M$ (1988) Croissance du taillis de châtaignier en France : premiers résultats. Ann Sci For 45, 265-274

Bédéneau M, Pagès L (1984) Étude des cernes d'accroissement ligneux du système racinaire d'arbres traités en taillis. Ann Sci For 41, 59-68

Blake TJ, Raitanen WE (1981) A summary of factors influencing coppicing. International Energy Agency, Rep. NE 1981 22, $24 \mathrm{p}$

Bonneau M (1988) Le diagnostic foliaire. Rev For Fr 40 (sp), 19-28

Cabanettes A (1989) Une méthode pour l'estimation de la biomasse ligneuse aérienne dans les jeunes taillis. Acta Oecol Oecol Appl $10,65-80$

de Champs J (1972) La production des taillis de châtaignier. AFOCEL, CR d'activité, 221-274

Chartier M (1987) The french biomass programme. In : Biomass for energy and industry. 4th EC Conf (Grassi G, Molle JF, Zibetta $H$, ed) Orléans, France, 1987/05/11-15. Elsevier Appl Sci, Londres, 19-35

Czapowskij MM, Safford LO (1979) Growth response to fertilizer in a young aspen-Birch stand. US Dep Agric For Serv Res Note NE$274,6 p$

Dacunha-Castelle D, Tomassone R (1975) Eléments de statistique non paramétrique. INRA Laboratoire de biométrie du CNRS, Jouy-enJosas (France), Doc 75/09, sp

Dagnélie P (1970) Théorie et méthodes statistiques. Applications agronomiques. Vol 2. Duculot, Gembloux (Belgique), $451 \mathrm{p}$

Dubois JM (1977) Essais sur taillis de Charme dans le Nord-Est et de la France. AFOCEL, CR d'activité 1977, 339-406
Duchaufour P (1970) Précis de pédologie. Masson, Paris, $481 p$

Evans J (1984) Sylviculture of broadleaved woodland. For Com Bull 62, Her Majesty's Stationery Office, Londres, $232 \mathrm{p}$

Evans $J(1986)$ Nutrition experiments in broadleaved stands : Il. Sweet chestnut and stored oak coppice. $Q J$ For Res 80, 95-104

Ferm A, Markkola A (1985) Nutritional variation of leaves, twigs and buds in Betula pubescens stands during season. Folia For 613, 1 28

Ferm A, Kauppi A, Rinne P (1982) The potential of birch for biomass production. Int Energy Agency For Planning Group $B$ Growth and production, Rapport JAB-34, $67 p$

Herbert MA (1983) The response of Eucalyptus grandis to fertilizing with nitrogen, phosphorus, potassium and dolomitic lime on a mispah soil series. South Afr For J 124, 4-12

Hocker HW (1982) Effects of thinning on biomass growth in young Populus tremuloides plots. Can J For Res 12, 731-737

Hubert M (1983) Amélioration des taillis par balivage intensif. Inst Dév For Paris, $200 \mathrm{p}$

Kauppi A, Rinne P, Ferm A (1988) Sprouting ability and significance for coppicing of dormant buds on Betula pubescens Ehrh stumps. Scand J For Res 3, 343-354

Kazarjan VO (1969) Le vieillissement des plantes supérieures. Nauka, Moscou, Trad fr, $194 \mathrm{p}$

Lanier L (1986) Précis de sylviculture. École nat génie rural, eaux et for Nancy, $468 p$

Mathey A (1931) Traité théorique et pratique des taillis. Vilaire, le Mans, $353 p$

Mattoon WR (1930) Growing Black Locust trees. US Dep Agric Farmers Bull, 1628, $13 \mathrm{p}$

Mroz GD, Frederick DJ, Jurgensen MF (1984) Site and fertilizer effects on northern hardwood stump sprouting. Can J For Res 15, 535-543

Pagès $L$ (1986) Lois de croissance en biomasse du taillis : le robinier dans le Val-de-Loire. Ann Sci For 43, 533-550

Parade A (1860) Cours élémentaire de culture des bois. Grimblot/Raybois, $4^{\theta}$ éd, Nancy, $699 \mathrm{p}$ 
Pardé J (1979) Biomasses forestières et énergie. Conférence prononcée à l'INAPG le 15 avril 1979. INRA CNRF (Nancy), Doc int 79/ 01, $34 \mathrm{p}$

Riedacker A (1979) Quelques réflexions d'ordre biologique et sociologique à propos d'une meilleure utilisation des ressources forestières françaises pour la production d'énergie. In : Bases biologiques ot sociologiques pour une utilisation rationnellle de la forêt pour la production d'énergie et de composés organiques, réunion UNESCO-MAB 6-11, mai $1979,6 p$

Riedacker A, Knockaert C, Zaidi A (1985) Production ligneuse des taillis d'eucalyptus : effet de la fréquence de coupe et de la densité de plantation. Ann Sci For 42, 39-52
Rullier-Bréval B (1985) Croissance d'un taillis de châtaignier après coupe. Étude au cours des trois premières années. Thèse Doct, Univ Paris-XI, $155 p$

Sabatier-Tarrago $C$ (1989) Production de taillis de châtaignier (Castanea sativa Mill) en relation avec les caractéristiques stationnelles. Thèse Doct, Paris-Sud Orsay, $250 \mathrm{p}$

Snedecor GW, Cochran WG (1957) Statistical Methods. $6^{\theta}$ édition. The lowa State Univ Press, Ames (USA), $649 \mathrm{p}$

Vaulot $G$ (1914) Le robinier faux-acacia. Histoire, description, culture, propriétés et utilisations. Baillière, Paris, $264 p$

Wenger KF (1953) The sprouting of sweetgum in relation to season of cutting and carbohydrate content. Plant Physiol 28, 35-49 